\title{
Space sustainability, advanced materials and micro/nanotechnologies for future life in outer Space
}

\author{
Loredana Santo $^{1}$ (D)
}

Received: 12 January 2022 / Accepted: 21 February 2022 / Published online: 4 March 2022

(c) The Author(s) 2022, corrected publication 2022

\begin{abstract}
The idea of living in Space is more and more a highly topical question. Orbital stations, the Moon, Mars are new possible lands for living, but to reach this goal, new advanced materials should be investigated, and micro/nano technologies exploited considering a sustainable development. The building of spacecraft, habitat modules and spacesuits for astronauts and Space tourists for going to the deep space needs high performance and shielding materials and micro/nanoengineering. In this communication, the new concept of Space sustainability is highlighted, the achievement in advanced sustainable material design and testing in Space and the promising use of micro/nanotechnologies for space applications are discussed.
\end{abstract}

Keywords Space sustainability $\cdot$ Shape memory polymer composite $\cdot$ Cosmic ray shielding $\cdot$ Space micro/nanotechnologies

The concept of sustainable development is well known and defined as "development that meets the needs of the present without compromising the ability of future generations to meet their own needs" [1]. Moreover, 17 Sustainable Development Goals (SDGs) or Global Goals were set up in 2015 by the United Nations General Assembly and are intended to be achieved by the year 2030 . They have been designed to be a "blueprint to achieve a better and more sustainable future for all" [2].

All that can be extended to the possible life in Space and firstly connected to the "Space colonization".

Remembering that the pillars of sustainability are mainly three: social, environmental, and the economic one, if we think about Space, Space sustainability can be defined in different ways. It is surely to make good educational courses in order to spread the concept of sustainable development in/for Space, to have the possibility to colonize Moon, Mars for future scenario for ensuring life when the Earth will become harsh, to find solutions for the survival of Human in Space, to make researches in Space for better life on Earth, to enhance telecommunication, to maintain the Space clean and save energy, to connect visions and cultures for future

Loredana Santo

loredana.santo@uniroma2.it

1 Department of Industrial Engineering, University of Rome Tor Vergata, Via del Politecnico 1, 00133 Rome, Italy life in outer space, to develop Space law, policy and regulations, etc.

Safety of Space (Space cleaning, Space weather...), Education, Scientific and Technical Research for in-Space Sustainability (educational training courses, in-Space manufacturing, debris removal, Space biomedicine, connecting visions/cultures...), Space Law, Policy and Regulation, Industry and Space Economy need to be investigated.

Therefore, Space sustainability is "Ensuring that all humanity can continue to use outer space for peaceful purposes and socioeconomic benefit now and in the long term. This will require international cooperation, discussion, and agreements designed to ensure that outer Space is safe, secure, and peaceful", as defined by the United Nations Office for Outer Space Affairs (UNOOSA) [3] and can be examined by different point of view with a multidisciplinary approach. Consequently, it is strategic to integrate sustainability principles into existing policies and procedures to foster awareness, approaches, and actions for a more sustainable world and Space.

In this frame, micro/nanotechnologies, innovative materials, and their manufacturing in the Space environment play a fundamental role. In fact, they could allow to reach planets far from the Earth and possibly life there. They must be used to build spacecraft, habitat modules, to clean Space, to store energy, and also to shield the astronauts and Space tourists for going to the deep Space. In this context, Shape Memory Polymer Composites (SMPCs) and Cosmic Ray 
Shielding (CRS) materials are very interesting for their properties and behaviour. They are sustainable materials not for their chemical nature, but for their functionalities. In fact, SMPCs can clean Space by removing space debris and allow the use of solar energy by deploying solar panels and solar sails. Moreover, as tested, they are low weight and durable in the Space environment. The SMPCs can be made with composite plies and shape memory interlayer. Commercial CFR prepregs for autoclave moulding of aeronautical parts have been used for samples fabrication (HexPly/M49/42\%/ CHS-3 K by Hexcel). These prepregs are $0 / 90$ fabrics with high performance epoxy matrix, not suitable for shape memory (SM) applications. For this reason, the functional SM behaviour was added to the composite laminates by using Scotchkote $206 \mathrm{~N}$ by $3 \mathrm{M}$, an uncured epoxy resin in the form of green fine powder. This powder has been used as interlayer during composite lamination and to produce foams by solid state foaming. The SM behaviour of this epoxy resin as interlayer or foam and of SMPCs was fully evaluated in several previous works of the author $[4,5]$.

The CRS materials are instead new shielding material for spacesuits (i.e. flexible) and possibly spacecraft materials. The CRS materials are a combination of low-density polyethylene (LDPE) film and inorganic particles (Samarium cobalt ( $\mathrm{Sm}-\mathrm{Co}$ ) and boron nitride (BN) powders).

The active layer consists of magnetic particles (Sm-Co) which can provide a small local magnetic field. That is a simple way to produce hybrid active passive shields. It is not expected that the small magnetic field is able to deflect solar events. It is under question if a local magnetic field is able to change the absorption of cosmic rays into the matrix. Moreover, it is not excluded that secondary emissions could be detrimental, and some additional shields must be added for this reason. In fact, Samarium has a very high mass and secondary emissions are expected to be a threat. Therefore, $\mathrm{BN}$ in the shape of particles has been used between two
LDPE sheets for contributing to reduce secondary emissions. CRS samples were manufactured in the correct stacking sequence which consisted, from the top to the bottom, in an unfilled LDPE layer, a coupled LDPE layer with BN powder, a coupled LDPE layer with Co-Sm powder and a last unfilled LDPE layer. The two internal coupled layers were rotated to have four different stacking sequences in the same CRS sample [6].

SMPCs and CRS materials have been successfully tested by the author in different Space missions for evaluating their behaviour in microgravity $[7,8]$ and the harsh space environment [6,9]. Recent experiments are flying as part of NASA Glenn Research Center's Polymers and Composites Experiment (PCE) and Polymers and Composites Experiment-2 (PCE-2) in the MISSE 9 and MISSE 10 missions, respectively [6]. They include 3 samples on MISSE-9 and 4 samples on MISSE-10 being flown in ram, wake, zenith or nadir orientations for Space environmental durability assessment. Figure 1 shows two prototypes respectively of CRS and SMPC samples for the MISSE-9 Polymers and Composites Experiment.

In the MISSE 9, in the case of SMPC, the experiments aim to show if sample recovery can happen on orbit, because of direct heating from the Sun or heat transfer from the surrounding platform. In this case, the window in Fig. 1b should close. In addition, the experiment aims to estimate the aging effect of the Space exposure in terms of mass loss, material degradation (cross-linking, chain polymer break, delamination, and embrittlement) and, above all, loss of performances (including shape memory behaviour). Such information will be fundamental for applying these materials in outer Space. In fact, the results of the SMPC samples will contribute to the development of self-deployable structures and grabbing systems.

In the case of CRS, the Space experiments want to test 4 different shielding generated by the stacking sequence
Fig. 1 Prototypes of CRS and SMPC

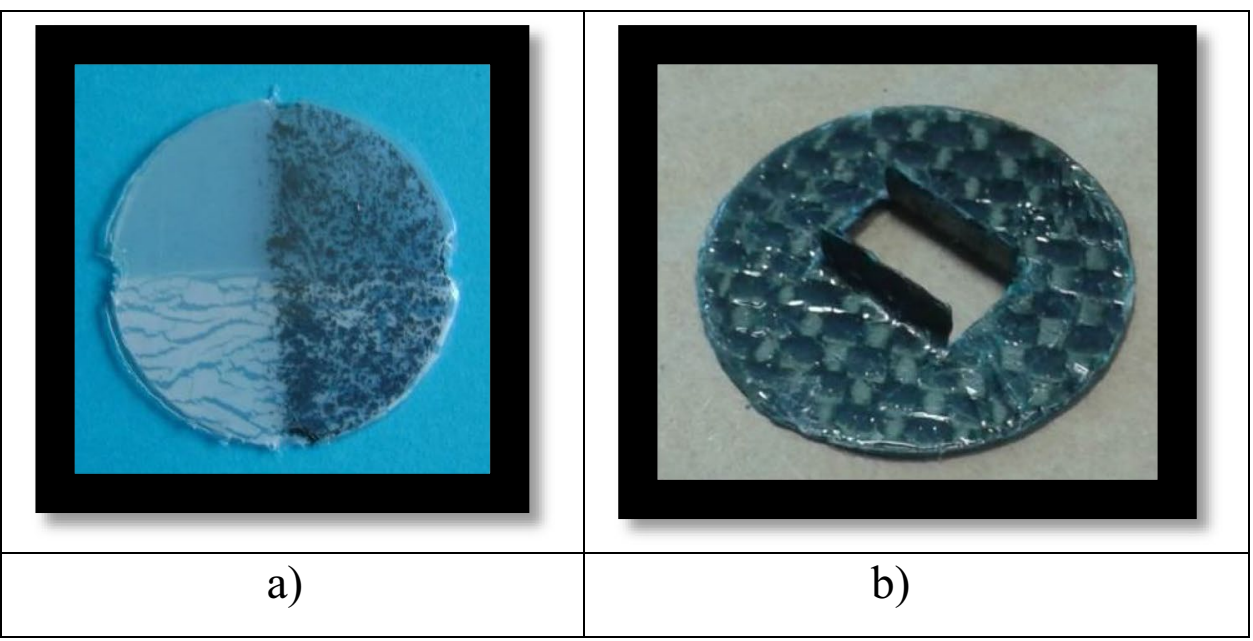


(evident in Fig. 1a). Such experiments are a first step toward the definition of new shielding materials mainly for space suits (i.e. flexible) but possibly also for spacecraft. New materials, new systems are necessary not only for Space applications and colonization. Being a harsh environment, materials tested in the Space may have Earth applications.

Figure 2 shows the CRS and SMPC samples on MISSE 9. In particular, in (a) Pre-flight photograph of the MISSE-9 wake deck, and in (b) On-orbit image of the wake samples after 7 months of space exposure are respectively shown. Figure 3 shows the SMPC sample on MISSE 9: (a) Pre-flight photograph of the MISSE-9 zenith deck, and (b) On-orbit image of the zenith SMPC sample (top right) after 7 months of space exposure [6].

The effect of the space exposure after 7 months on the International Space Station's MISSE-Flight Facility is well evident: degradation of the CRS sample and partial recovery of the SMPC sample but only in the zenith orientation. Indepth analyses are currently underway.

Anyway, advanced materials should be coupled with micro/nanotechnologies for promoting the Space sustainability. In fact, the latter could contribute to a significant reduction in the cost of access to Space, profiting from microsystems' recognised advantages and could make possible certain types of missions and applications, by virtue of their volume/mass/power reduction potential [10].

Nanomaterials, nanosensors and nano-instrumentation, microcraft, micro/nano robots, nanomanufacturing are just

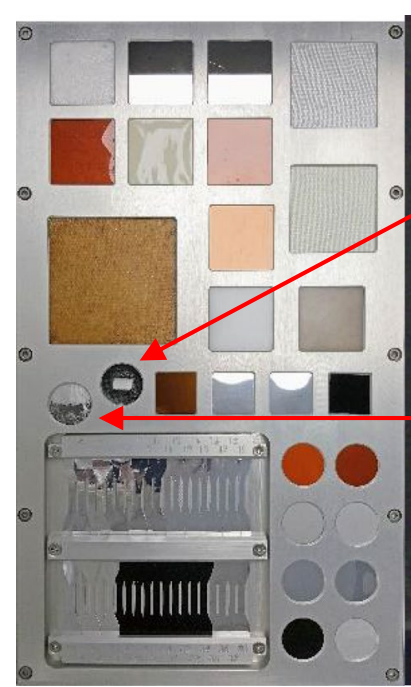

a)

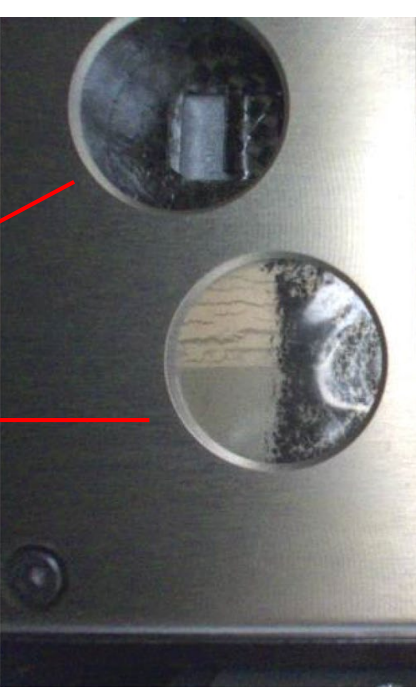

b)
Fig. 2 CRS and SMPC samples on MISSE 9: (a) Pre-flight photograph of the MISSE-9 wake deck, and (b) On-orbit image of the wake samples after 7 months of space exposure. Photo credit: Aegis Aerospace and NASA

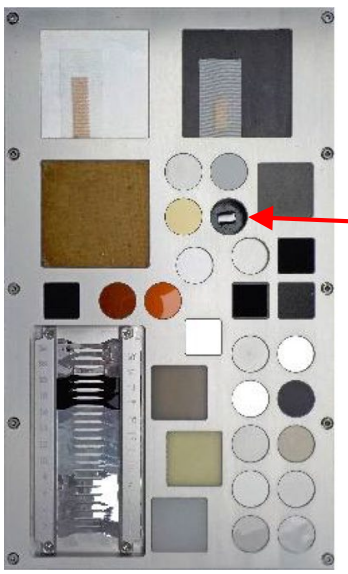

a)

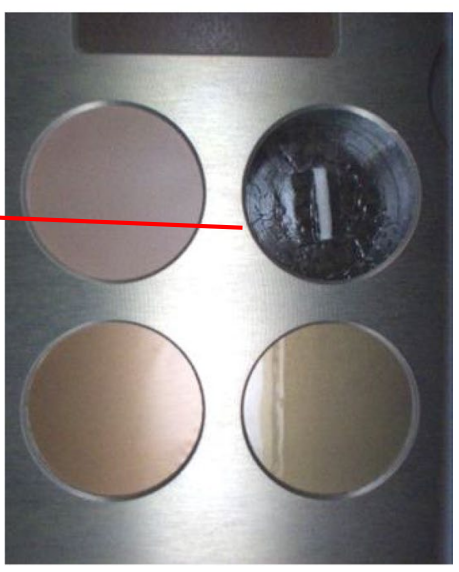

b)
Fig. 3 SMPC sample on MISSE 9: (a) Pre-flight photograph of the MISSE-9 zenith deck, and (b) On-orbit image of the zenith SMPC sample (top right) after 7 months of space exposure. Photo credit: Aegis Aerospace and NASA

some promising areas of interest. Among these, miniaturization and micro/nanotechnology in space robotics are object of interesting research [11] because robots can be used for different purposes in the Space environment.

At the end, advanced materials and micro/nanotechnologies are the premise for reaching new planets and allow life in Space but only interdisciplinary interconnection between researchers in different fields, including engineers, scientist, clinicians, etc. can promote and make effective Space sustainability for long-term missions and future life in outer Space.

Acknowledgements I am very grateful to the NASA Flight Opportunities program, the International Space Station Program Office, Aegis Aerospace and Nasa Glenn Research Center for making the mentioned flight opportunities possible.

Funding Open access funding provided by Università degli Studi di Roma Tor Vergata within the CRUI-CARE Agreement.

\section{Declarations}

Conflict of interest The author declares no competing interests.

Open Access This article is licensed under a Creative Commons Attribution 4.0 International License, which permits use, sharing, adaptation, distribution and reproduction in any medium or format, as long as you give appropriate credit to the original author(s) and the source, provide a link to the Creative Commons licence, and indicate if changes were made. The images or other third party material in this article are included in the article's Creative Commons licence, unless indicated otherwise in a credit line to the material. If material is not included in the article's Creative Commons licence and your intended use is not permitted by statutory regulation or exceeds the permitted use, you will need to obtain permission directly from the copyright holder. To view a copy of this licence, visit http://creativecommons.org/licenses/by/4.0/. 


\section{References}

1. World Commission on Environment and Development. Our Common Future. Oxford University Press. Oxford. UK, 1987 quoted in Pope J, Annandale D, Morrison-Saunders A. Conceptualizing sustainability assessment. Environmental Impact Assessment Review, 2004;24:595-616.

2. https://www.un.org/sustainabledevelopment/sustainable-devel opment-goals/

3. Guidelines for the Long-term Sustainability of Outer Space, https://www.unoosa.org/res/oosadoc/data/documents/2018/aac 1052018crp/aac_1052018crp_20_0_html/AC105_2018_CRP20E. pdf, 2018.

4. Santo L., Shape memory polymer foams, 2016, Progress in Aerospace Sciences, Volume 81, Pages $60-65$.

5. F. Quadrini, L. Iorio, D. Bellisario and L. Santo, Shape memory polymer composite unit with embedded heater, Smart Materials and Structures 30 (2021) 075009 (14 pp).

6. Santo L., Quadrini F., Bellisario D., De Groh K. K., Shape Memory Polymer Composites and Cosmic Ray Shielding Materials in Open Space, Proceedings of the Applied Space Environments Conference (ASEC), May 13-17, 2019, Los Angeles.

7. L. Santo, F. Quadrini, G. Mascetti, F. Dolce, V. Zolesi, Mission STS-134: results of shape memory foam experiment. Acta Astronautica 91, 333-340 (2013)

8. L. Santo, F. Quadrini, P.L. Ganga, V. Zolesi, Mission BION-M1: results of Ribes/Foam2 experiment on shape memory polymer foams and composites. Aerospace Science and Technology 40, 109-114 (2015)

9. Kim K. de Groh, Bruce A. Banks, MISSE-Flight Facility, Polymers and Composites Experiment 1-4 (PCE 1-4), NASA/ TM-20205008863, February 2021.

10. A.Martinez de Aragon, Future Applications of Micro/Nano Technologies in Space Systems, European Space Agency, https://www. esa.int/esapub/bulletin/bullet85/mart85.htm

11. Papadopoulos E., Paraskevas I.,Flessa T., 2013, Miniaturization and micro/nanotechnology in space robotics, Nanorobotics: Current Approaches and Techniques Pages 69 - 921. 